bioRxiv preprint doi: https://doi.org/10.1101/2022.01.23.477418; this version posted January 24, 2022. The copyright holder has placed this preprint (which was not certified by peer review) in the Public Domain. It is no longer restricted by copyright. Anyone can legally share, reuse, remix, or adapt this material for any purpose without crediting the original authors.

\title{
1 Proteostasis is differentially modulated by inhibition of translation initiation or
}

2 elongation

3

4

$5 \quad$ Khalyd J. Clay, Yongzhi Yang and Michael Petrascheck*

6

7 Department of Molecular Medicine, The Scripps Research Institute, La Jolla, United States; Department of

8 Neuroscience, The Scripps Research Institute, La Jolla, United States

9

*For correspondence: pscheck@scripps.edu 


\section{Abstract}

Recent work has revealed an increasingly important role for mRNA translation in maintaining proteostasis. Inhibiting translation protects from various proteostatic insults, including heat, expression of aggregation-prone proteins, or aging. However, multiple studies have come to differing conclusions about the mechanisms underlying the protective effects of translation inhibition. Here, we systematically lower translation either by pharmacologically inhibiting translation initiation or elongation and show that each step activates distinct protective responses in Caenorhabditis elegans. Targeting initiation triggers an HSF-1 dependent mechanism that protects from heat and age-associated protein misfolding but not from proteotoxicity caused by proteasome dysfunction. Conversely, targeting elongation triggers an HSF-1 independent mechanism that protects from heat and proteasome dysfunction but not from age-associated protein aggregation. Furthermore, while inhibiting translation initiation increases lifespan in wild-type worms, inhibiting translation elongation only extends lifespan when the animals exhibit preexisting proteotoxic stress-either as a result of aggregationprone protein expression or hsf-1 deficiency. Together our findings suggest that organisms evolved complementary mechanisms that the mRNA translation machinery can trigger to restore proteostasis.

\section{Introduction}

Protein synthesis is a highly regulated process involving the precise orchestration of many chaperones, cofactors, enzymes, and biomolecular building blocks. It is critical at every level of the life cycle from development through aging and is central to stress adaptation (Higuchi-Sanabria et al., 2018). Protein synthesis largely determines the folding load on the proteostasis network, which regulates protein production, folding, trafficking, and degradation to maintain a functional proteome. The imbalance of the proteostasis network caused by age-associated stress or acute environmental insults leads to misfolding of proteins and the accumulation of aggregates, and eventually to disease (Balch et al., 2008).

A substantial body of work has revealed that the protein synthesis machinery directly participates in protein folding or aggregation. For example, in mice, point mutations in specific tRNAs or components of the ribosomal quality control (RQC) pathway can lead to protein aggregation and neurodegeneration (Chu et al., 2009; Nedialkova and Leidel, 2015; Nollen et al., 2004; Vo et al., 2018; Yonashiro et al., 2016). Similarly, early RNAi screens in the nematode $C$. elegans identified several ribosomal subunits whose knockdown increased aggregation of polyglutamine (polyQ) proteins (Nollen et al., 2004). These findings highlight the importance of translation in protein misfolding.

However, subsequent studies reveal a more intricate role of translation in protein aggregation. Depending on how translation is modulated can lead to either decreased or increased protein aggregation. For example, RNAimediated knockdown of translation initiation factors increase lifespan, improve proteostasis, and reduce protein aggregation in C. elegans (Balch et al., 2008; Lan et al., 2019; McQuary et al., 2016; Rogers et al., 2011). Similarly, work in yeast and cell culture shows that pharmacological inhibition of translation prior to a heat shock prevents proteins from aggregating (Choe et al., 2016; Medicherla and Goldberg, 2008; Riback et al., 2017; Xu et al., 2016). While studies in C. elegans, cell culture, and yeast agree that inhibition of translation reduces 
protein aggregation, their proposed underlying mechanisms differ. Overall, the proposed mechanisms can be categorized into two broad models on how lowering translation reduces protein aggregation.

The first model, referred to as the selective translation model, proposes that inhibition of translation is selective. In the selective translation model, the increased availability of ribosomes leads to differential translation of mRNAs coding for stress response factors and thus to increased folding capacity (Lan et al., 2019; McQuary et al., 2016; Rogers et al., 2011; Seo et al., 2013). In general, studies proposing a version of the selective translation model show that inhibiting translation requires HSF-1 to reduce protein aggregation (Tye and Churchman, 2021). In the selective translation model, protein aggregation is reduced by an HSF-1 dependent active generation of folding capacity to remodel the proteome.

The second model, referred to as the reduced folding load model, proposes that newly synthesized proteins are the primary aggregation-prone species of proteins. Therefore, newly synthesized proteins constitute the most significant folding load on the proteostasis machinery. Inhibition of translation reduces the concentration of newly synthesized proteins and thus the load on the folding machinery. In contrast to the selective translation model, which proposes selective protein synthesis of HSF-1 dependent stress response factors, the reduced folding load model does not depend on specific factors but generates folding capacity by reducing the overall folding load. A fundamental problem comparing previous studies has been their use of different model organisms, different proteostatic insults, and modes of inhibition, each of which could potentially account for the varied conclusions.

In this study, we systematically compared these two models in C. elegans using pharmacological agents to block various steps along the protein production cycle. We characterize how lowering translation protects $C$. elegans from proteotoxic insults such as proteasome inactivation, heat shock, and aging. We find evidence for both the selective translation and the reduced folding load models. Our data reveal that the step inhibited in mRNA translation dictates which of the two protective mechanisms is activated. Furthermore, we show that the two mechanisms are complementary in protecting from proteostatic insults suggesting that a cell may activate one over the other depending on the proteostatic stress. Overall, we provide evidence that elongation inhibitors ameliorate ongoing toxicity by reducing folding load. In contrast, initiation inhibitors elicit an active mechanism that prevents damage by remodeling the proteome dependent on HSF-1 and a likely increase in protein turnover via the proteasome. 
A

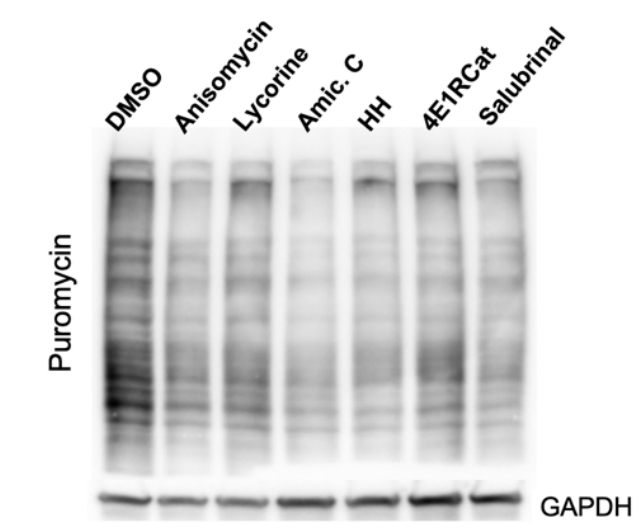

C

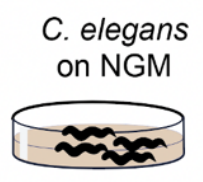

Days of adulthood

D

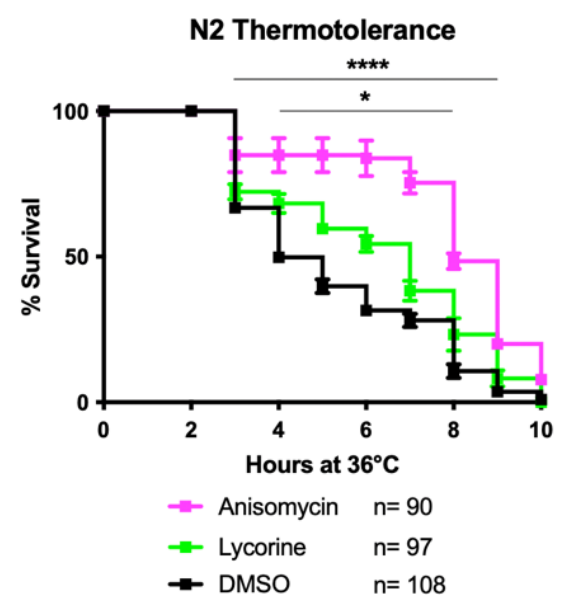

E
B

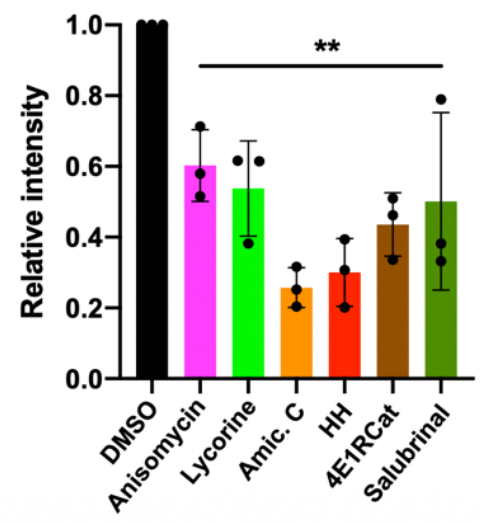

Hourly scoring

\section{Pretreatment 4 Days Heat Shock}

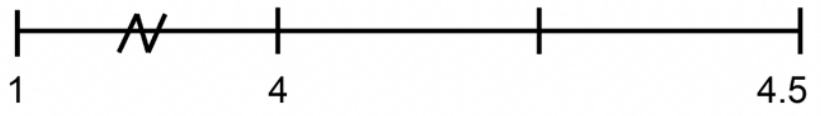

N2 Thermotolerance

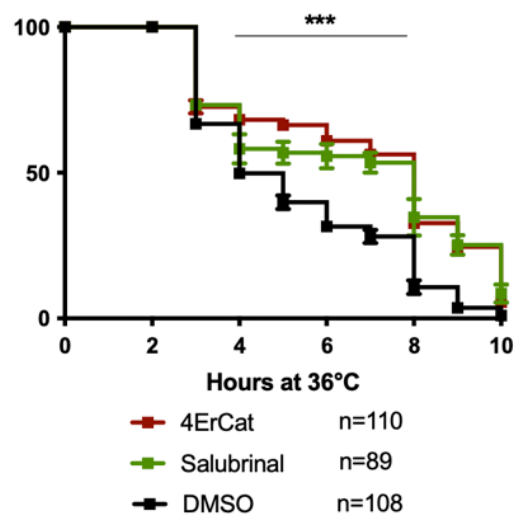

Figure 1: Translation inhibitors improve thermotolerance

A) Monitoring changes in protein synthesis using the SUnSET method. C .elegans were treated with solvent (DMSO) or the indicated inhibitors $(100 \mu \mathrm{M})$ for 12 hours, followed by a 4-hour puromycin incorporation. Worms were lysed, and protein extracts were run on SDS-PAGE gels, followed by staining with puromycin antiserum. GAPDH was used as a loading control.

B) Quantification of three independent SUnSET experiments. Significance was determined by one-way ANOVA with Dunnett's multiple comparisons test where $* *=p \leq 0.01$ Error bars indicate mean \pm SD from three independent trials.

C) Day 1 adult wild-type (N2) animals were treated for 4 days, then transferred to NGM plates. Animals were then subjected to a constant, non-permissive temperature of $36^{\circ} \mathrm{C}$ and scored alive/dead every hour by movement following a gentle tap.

D) Graph shows survival as a function of hours at $36^{\circ} \mathrm{C}$ of day 4 adult wild-type (N2) animals. The animals were pretreated with either DMSO (solvent control), anisomycin or lycorine. Data are displayed as mean \pm SEM from three independent trials and * $=p \leq 0.05$ and ${ }^{* * * *}=p \leq 0.0001$ by row-matched two-way ANOVA with Šídák multiple comparisons test.

E) Same as in Figure 1D but showing initiation inhibitors 4E1RCat and salubrinal. Data are displayed as mean \pm SEM from three independent trials and ${ }^{* * *}=p \leq 0.0002$ by row-matched two-way ANOVA with Šídák multiple comparisons test. 


\section{Results}

\section{Translation inhibitors improve thermotolerance}

We first set out to identify suitable pharmacological initiation and elongation inhibitors by screening a series of translation inhibitors (Table 1) for their ability to inhibit protein synthesis in C. elegans (Dmitriev et al., 2020). To monitor protein synthesis, we employed SUrface SEnsing of Translation (SUnSET) (Arnold et al., 2014). In this method, translating ribosomes incorporate puromycin into newly synthesized proteins. The level of puromycin incorporation

\begin{tabular}{|c|c|c|}
\hline Name & Inhibits & Target \\
\hline 4E1RCat & Initiation & elF4G \\
\hline Salubrinal & Initiation & elF2 $\alpha$ \\
\hline Anisomycin & $\begin{array}{l}\text { Peptidyl transferase } \\
\text { center }\end{array}$ & A-site \\
\hline Lycorine & $\begin{array}{l}\text { Peptidyl transferase } \\
\text { center }\end{array}$ & A-site \\
\hline Homoharringtonine & Elongation/Initiation & A-site \\
\hline Amicoumacin C & Translocation & E-site \\
\hline
\end{tabular}
serves as a quantitative measure for translation and is detected by Western Blotting using an anti-puromycin monoclonal antibody. All six molecules (Table 1) reduced puromycin incorporation relative to DMSO controls to varying degrees (Figure $1 \mathrm{~A} \& \mathrm{~B}$ ). Based on the similar inhibitory effects, we selected the two initiation inhibitors-4E1RCat and salubrinal-and the two elongation inhibitors-anisomycin and lycorine-to investigate how chemically targeting two steps of the translation cycle will affect stress-induced protein aggregation.

We chose thermal stress as the first stressor to induce protein aggregation and asked if both initiation and elongation inhibitors improve stress resistance. The animals were treated with either of the four inhibitors on day 1 of adulthood, and after 72 hours, treatment moved to a non-permissive temperature of $36^{\circ} \mathrm{C}$ (Figure 1C). Hourly monitoring revealed that all four molecules significantly improved the survival of N2 animals (Figure 1D \& E). These data show that both the inhibition of translation initiation and elongation protect from thermal stress.

\section{Initiation and elongation inhibitors protect from thermal stress by HSF-1 dependent and independent} mechanisms, respectively

We next asked if translation inhibitors require the canonical heat shock response (HSR) controlled by the transcription factor HSF-1 to protect from thermal stress. Previous work by us and others resulted in contradictory findings on whether protection from heat by translation inhibition depends on HSF-1 (Seo et al., 2013; Solis et al., 2018; Zhou et al., 2014). Therefore, to test if translation inhibition protects from thermal stress in an HSF-1 dependent or independent manner, we repeated the thermotolerance assay in HSR-deficient $h s f-$ 1(sy441) mutants (Figure 2A). Only the elongation inhibitors anisomycin and lycorine protected $h s f-1$ (sy441) from HS-induced proteotoxicity (Figure 2B). The initiation inhibitors 4E1RCat and salubrinal did not (Figure 2C). These results were surprising as they showed that different modes of translational inhibition activate distinct protective proteostasis mechanisms. However, this also reconciles previous contradictions as different groups inhibited translations using inhibitors with specificity for either step. 
45 To confirm the ability of elongation inhibitors to protect from thermal proteotoxicity through improved 46 proteostasis broadly, we conducted sequential detergent extractions to biochemically isolate and quantify 47 soluble and insoluble proteins in wild-type (N2) animals (David et al., 2010; Reis-Rodrigues et al., 2012; Simonsen 48 et al., 2008). Following a 2 hour HS at $36^{\circ} \mathrm{C}$, we observed a substantial increase in insoluble protein compared 49 to non-heat shocked controls. Furthermore, pre-treatment with the elongation inhibitor anisomycin suppressed 50 the increase in protein insolubility (Figure 2D and E) consistent with the observed thermal protection. Using the $51 h s p-16.2:: G F P$ reporter strain, we further confirmed that anisomycin treatment does not activate the HSR, either 52 alone or in combination with a heat shock (see below, Fig. 4E). These results reveal that translation initiation 53 inhibitors trigger an HSR dependent mechanism to protect from thermal stress, while translation elongation 54 inhibitors trigger an HSR independent mechanism. We concluded that different modes of translational inhibition 55 protect the proteome by genetically distinct mechanisms. 
A

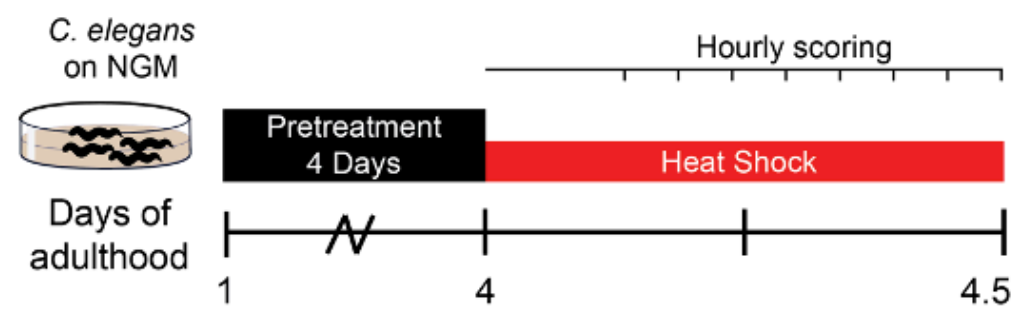

B
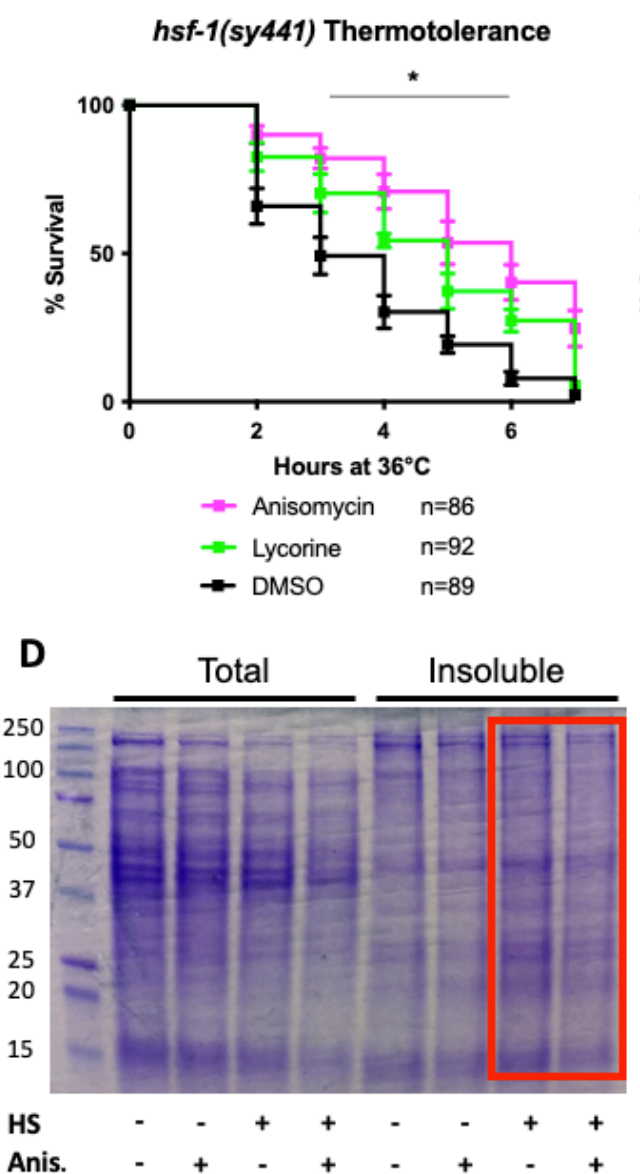

C

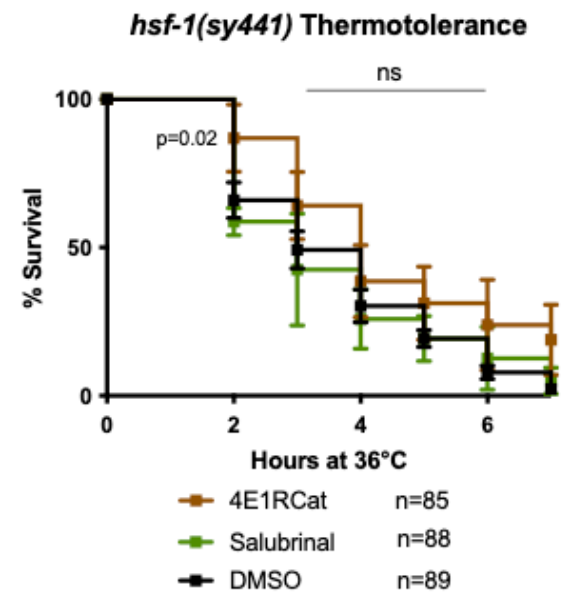

E

N2 Day 2 Extracts

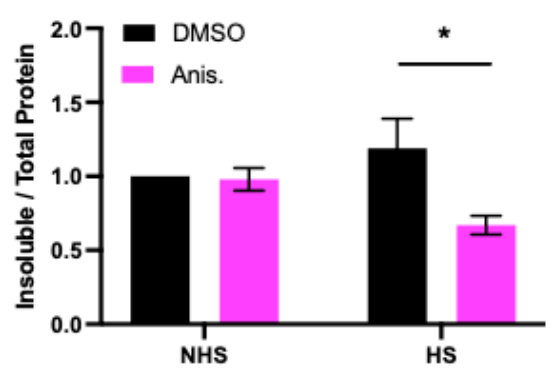

Figure 2: Initiation but not elongation inhibitors depend on HSF-1 to protect from thermal stress

A) Day 1 adult $h s f-1$ (sy441) animals were treated for 4 days, then transferred to NGM plates. They were then subjected to a constant, non-permissive temperature of $36^{\circ} \mathrm{C}$ and scored alive/dead every hour by movement following a gentle tap.

B) Thermotolerance assay: Graph shows survival as a function of hours at $36^{\circ} \mathrm{C}$ of day 4 adult $h s f-1$ (sy441) animals pretreated with either anisomycin or lycorine. Data show the mean \pm SEM from three independent trials and $*=p \leq 0.05$ by row-matched two-way ANOVA with Šídák multiple comparisons test.

C) Same as in Figure 2B but showing initiation inhibitors 4E1RCat and salubrinal. Data are displayed as mean \pm SEM from three independent trials and $*=p \leq 0.05$ by row-matched two-way ANOVA with Šídák multiple comparisons test.

D) Representative SDS-PAGE gel stained with the protein stain Comassie Blue for visualization. Anisomycin (Anis.) reduces the proportion of detergent-insoluble protein following a 2 hour HS of N2 animals. Proteins were detergent extracted, ultracentrifuged, and the insoluble pellet was resuspended in $8 \mathrm{M}$ urea before running on the gel.

E) Quantification of 4 separate extractions shows anisomycin significantly reduces HS-induced aggregation in wild-type N2 animals. Gels were stain with Sypro Ruby. Data are displayed as mean \pm SEM and * $=p \leq 0.05$ by two-tailed students t-test. 
A

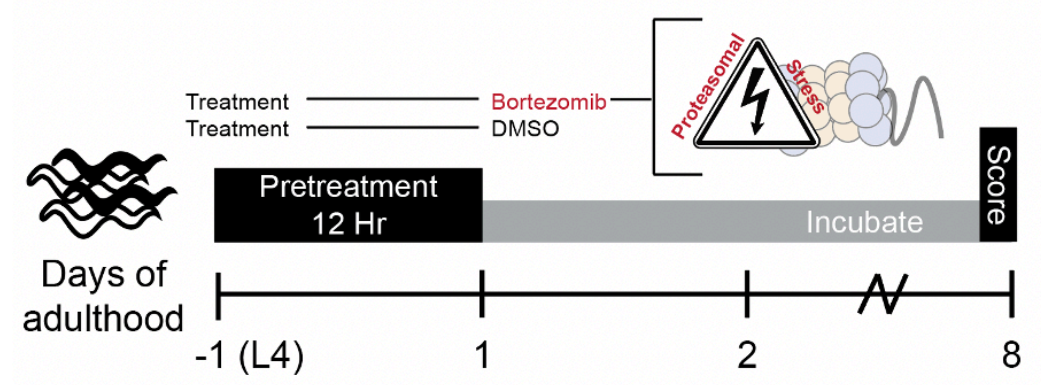

C

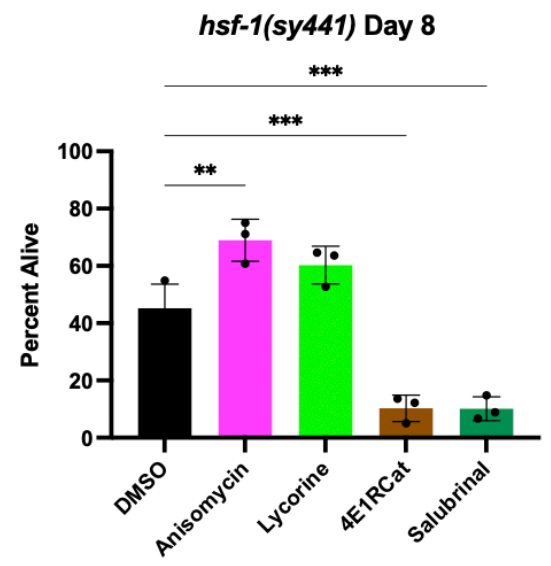

F

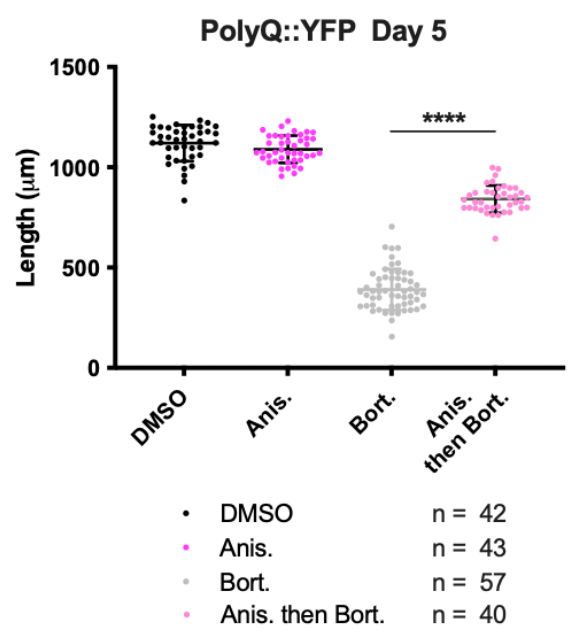

G

DMSO
B

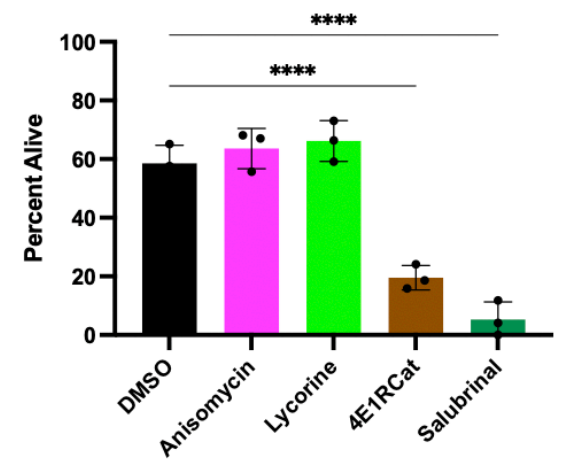

E
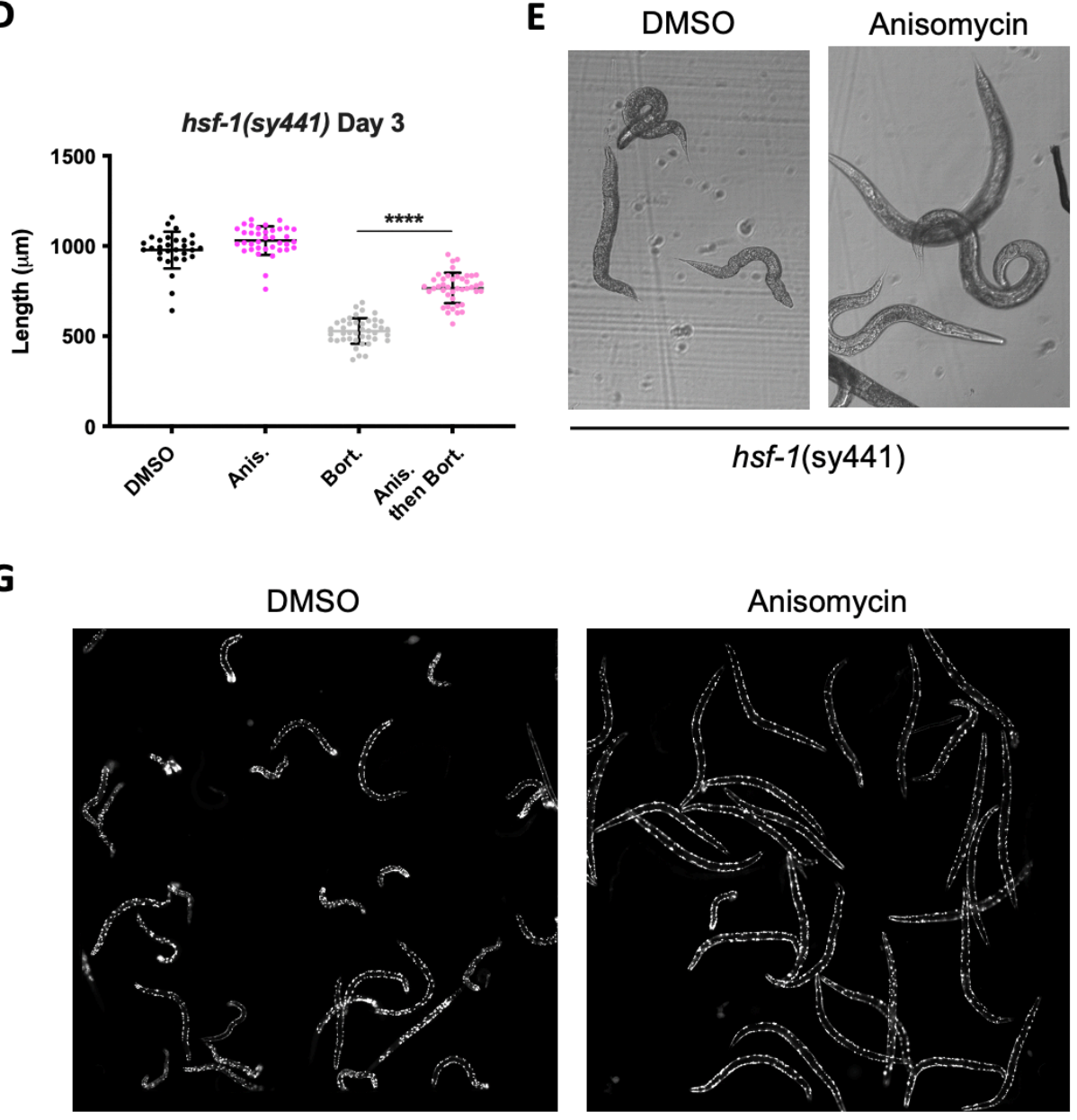

Q35::YFP

Figure 3: Elongation inhibitors protect from proteasomal stress independent of $h s f-1$

A) Experimental strategy for treating animals with translation inhibitors followed by inhibition of the 20S proteasome by bortezomib. Worms are pretreated for 12 hours with DMSO or indicated inhibitors, followed by bortezomib (75 $\mu \mathrm{M})$ treatment. The animals were then incubated with the combined treatment for 8 days and scored as alive/dead based on movement.

B) Elongation inhibitor treatment improved morphological phenotypes (not shown) but did not protect from bortezomibinduced proteotoxicity in N2 animals. Initiation inhibitor treatment enhanced toxicity of proteasomal stress. Data are displayed as mean \pm SD and $* * * *=p<0.0001$ by one-way ANOVA.

C) In $h s f-1$ (sy441) animals, elongation inhibitors protected from bortezomib-induced proteotoxicity while initiation inhibitors continue to sensitize worms to the resulting proteotoxicity. Data are displayed as mean \pm SD and ${ }^{* *}=p<0.01$ by one-way ANOVA. 
D) Measured length of $h s f-1$ (sy441) worms at day 3 of adulthood. Anisomycin treatment almost completely rescued the sma phenotype induced by bortezomib. Data are displayed as mean $\pm S D$ and $* * * *=p<0.0001$ by a two-tailed unpaired t-test.

E) Representative brightfield images of day 3 hsf-1(sy441) animals treated with the indicated compounds as outlined in figure 5A. Anisomycin pre-treatment prevented the sma phenotype observed to be caused by proteasomal inhibition.

F) Measured length of PolyQ worms at day 5 of adulthood. Anisomycin treatment almost entirely rescued the small (sma) phenotype induced by bortezomib. Data are displayed as mean $\pm S D$ and $* * * *=p<0.0001$ by a two-tailed unpaired t-test.

G) Representative fluorescent images PolyQ worms at day 5 of adulthood treated with the indicated compounds as outlined in Figure 5A. Bortezomib treatment caused the worms to contract into a sma and unc phenotype (left panel), and anisomycin pretreatment prevented these pathological phenotypes (right panel).

\section{Translational elongation, but not initiation inhibitors protect from proteasome dysfunction}

The data thus far suggests that inhibition of translation protects from thermal stresses by at least two mechanisms, one that involves HSF-1 and one that does not. We, therefore, investigated whether the protective mechanisms can be further distinguished by their capacity to protect from different proteostatic insults. One main proteostasis mechanism by which cells clear protein aggregates is the proteasomal system. The proteasomal system ubiquitinylates misfolded proteins by ubiquitin ligases to target them for degradation by the $26 \mathrm{~S}$ proteasome. Blocking proteasome degradation by bortezomib, a specific inhibitor of the $20 \mathrm{~S}$ subunit, results in the formation of protein aggregates and proteotoxic stress (Schrader et al., 2016).

Proteasomal stress induced by the treatment with bortezomib killed $\sim 40 \%$ of the wild-type N2 animals by day 8. Cotreatment of bortezomib with any of the two translation initiation inhibitors enhanced proteasome toxicity leading to over $80 \%$ of the animals dying, while cotreatment with elongation inhibitors improved morphology but had no effect on survival (Figure 3B). Thus, while initiation inhibitors protect from thermal stress, they sensitize the animals to proteasomal stress.

Next, we repeated the bortezomib-induced proteasomal stress survival experiments using strains with reduced protein folding capacity-either because of a mutation in $h s f-1$ or because expression of an aggregation-prone protein (PolyQ35::YFP). Treatment of hsf-1(sy441) mutant animals with bortezomib killed $\sim 60 \%$ of the $h s f$ 1(sy441) animals, a substantial increase in toxicity compared to N2. Cotreatment with translation initiation inhibitors again enhanced proteasome toxicity leading to over $80 \%$ of the animals to die. Cotreatment with elongation inhibitors, however, suppressed bortezomib toxicity and enabled about $70 \%$ of the hsf-1(sy441) animals to survive (Figure $3 \mathrm{C}$ ). This survival rate was close to what was seen in bortezomib-treated wild-type animals, suggesting that elongation inhibitors mostly rescue the $h s f-1$ (sy441) proteostasis phenotype. We also observed bortezomib-treated $h s f-1$ (sy441) animals to shrink in size (sma phenotype) and to become severely uncoordinated (unc phenotype). Treatment with anisomycin substantially rescued sma and unc phenotypes, almost completely reversing the animals back to normal size (Figure 3D \& F).

The AM140 strain expresses a stretch of 35 glutamine residues fused to YFP (PolyQ::YFP) in the muscle, which we used as a second model of reduced protein folding capacity. Expression of the aggregation-prone PolyQ stretch increases the protein folding load on the proteostasis system. Thus, its aggregation propensity acts as a sensor for protein folding capacity (Brignull et al., 2006; Moronetti Mazzeo et al., 2012). As expected, treatment of PolyQ::YFP animals with bortezomib caused extensive protein aggregation, a reduction in body size (sma phenotype), and an uncoordinated phenotype (unc phenotype). In addition, inhibition of translation initiation 
again exacerbated bortezomib toxicity but was not further quantified. In contrast, inhibition of elongation by

anisomycin almost entirely rescued both the sma and unc phenotypes revealing protection from proteasome toxicity in the context of reduced folding capacity (Figure $3 F \& G$ ).

We concluded that inhibition of translation initiation exacerbates bortezomib-induced proteasome stress, probably because its downstream protective mechanism heavily depends on proteasomal degradation (see discussion). We further concluded that elongation inhibition provided only limited direct protection from proteasomal stress since we did not see an increase in survival in wild-type animals. However, elongation inhibitors were highly protective from bortezomib toxicity in strains with reduced protein folding capacity, suggesting that elongation inhibitors protect by reducing the need for protein folding capacity by lowering the concentration of newly synthesized proteins.

\section{Translational elongation and initiation inhibitors protect from protein aggregation}

The ability of anisomycin to mitigate bortezomib toxicity in PolyQ::YFP animals suggested that translation elongation inhibitors free up folding capacity through the reduction of overall protein synthesis. To monitor folding capacity in live imaging, we developed a live imaging experimental procedure to examine how translation inhibition will dynamically affect protein aggregation in real-time (Figure 4A). After a 2 hour HS, the initially diffuse PolyQ signal gradually localized into puncta (Figure 4B, Video 1). Aggregation foci formation resulted from a redistribution of the YFP signal into aggregation foci as the total level of YFP fluorescent signal for a given animal remained constant (S1).

Only elongation inhibitors lycorine and anisomycin significantly reduced HS-induced polyQ aggregation, while the initiation inhibitors 4E1RCat and salubrinal did not (Figure 4C and S2). Pre-treatment with anisomycin and lycorine reduced the number of polyQ aggregates per worm. However, pre-treatment did not change the onset of aggregation, the rate of formation, or the final size of the aggregation foci (Morley et al., 2002) (Figure 4D). Furthermore, we found the effect of anisomycin or lycorine to be time-dependent. They strongly inhibited aggregation following a 12 hour preincubation period but less so following a 4-hour preincubation (S3). Starting treatment with anisomycin after the HS did not reduce the number of aggregation foci (not shown). These results suggest that anisomycin and lycorine reduce the early formation of aggregate foci but do not alter the dynamics once aggregation begins. Anisomycin dose-dependently decreased the activation of the HSR, as measured by the $h s p-16.2:: G F P$ reporter strain, confirming again that protection from aggregation is independent of $h s f-1$ (Figure 4E, S4). 
A

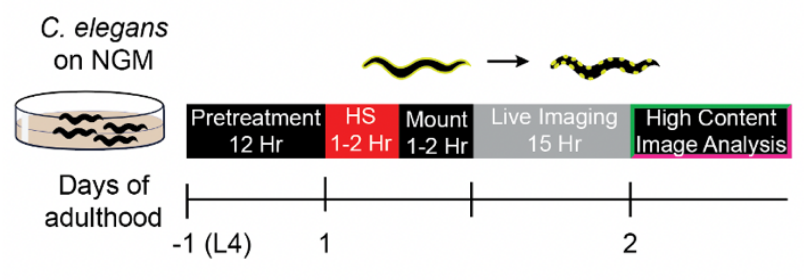

C

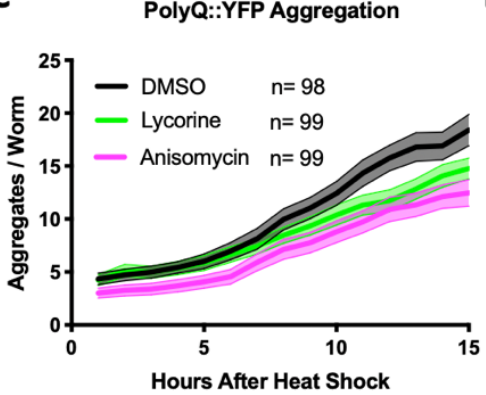

B

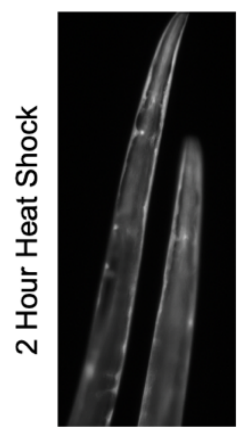

1 hour

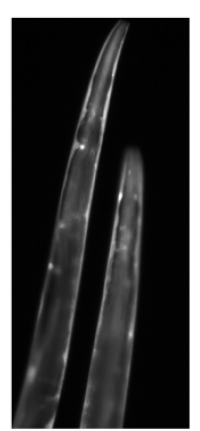

4 hour
8 hour

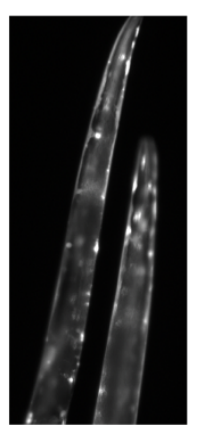

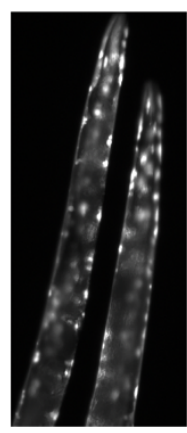

12 hour

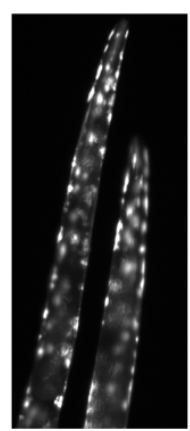

15 hour
E

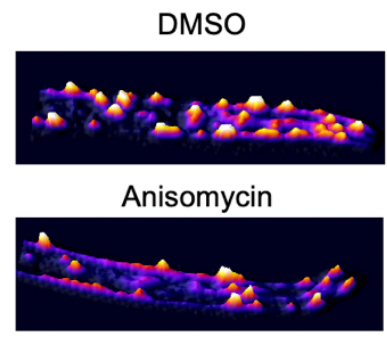

hsp16.2::GFP

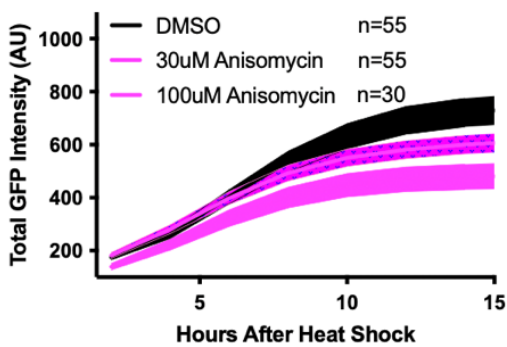

Figure 4: Elongation inhibition protects from heat shock-induced protein

A) Day 1 AM140 adult worms expressing the polyglutamine-YFP fusion protein (PolyQ::YFP) in their muscle were subjected to HS on NGM plates for 2 hours at $36{ }^{\circ} \mathrm{C}$ followed by a 1-2 hour mounting/immobilization procedure in 384 well plates and subsequent live imaging for 15 hours.

B) Fluorescent time-lapse images of two animals expressing the PolyQ::YFP fusion protein in their muscle. The animals were embedded in the hydrogel for immobilization. Following a 2-hour HS, animals were imaged over 15 hours; by 8 hours, the YFP signal began to localize into discrete puncta that persisted through the observation time. Overall fluorescence remained stable (see S2).

C) Graph shows the mean number of PolyQ aggregates per worm as a function of time following HS. C. elegans (PolyQ::YFP) were pretreated with lycorine, anisomycin $(100 \mu \mathrm{M})$, or DMSO. Lines indicate mean, and shading indicates $95 \% \mathrm{Cl}$.

D) (Top) Representative images of control and $100 \mu \mathrm{M}$ anisomycin treated PolyQ animals 15 hours after HS (Bottom). The representative images shown have been uniformly modified using the '3D Surface Plot' plugin in ImageJ to visualize aggregates. E) Dose-dependent suppression of HS-induced $h s p$-16.2::GFP reporter activation (Day 1 animals) after 12 hour pretreatment of anisomycin. $95 \% \mathrm{Cl}$, as indicated by shading. 
bioRxiv preprint doi: https://doi.org/10.1101/2022.01.23.477418; this version posted January 24, 2022. The copyright holder has placed this preprint (which was not certified by peer review) in the Public Domain. It is no longer restricted by copyright. Anyone can legally share, reuse, remix, or adapt this material for any purpose without crediting the original authors.

A

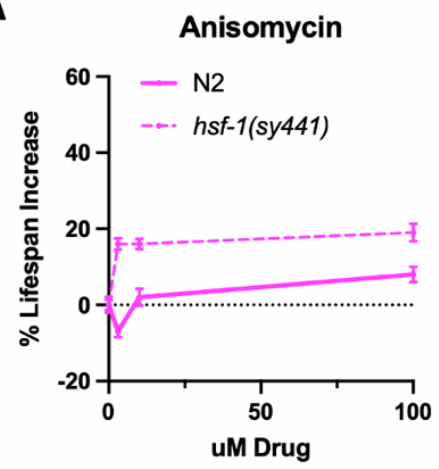

B

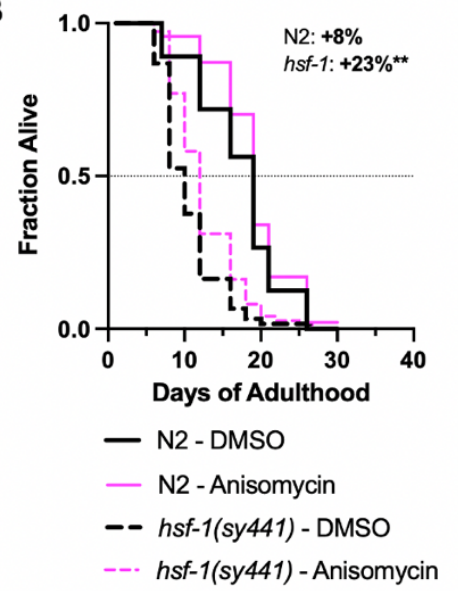

C

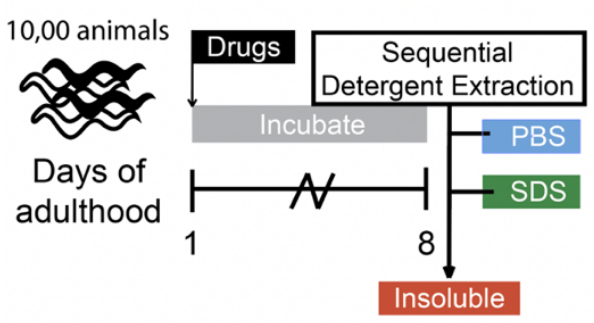

Lycorine
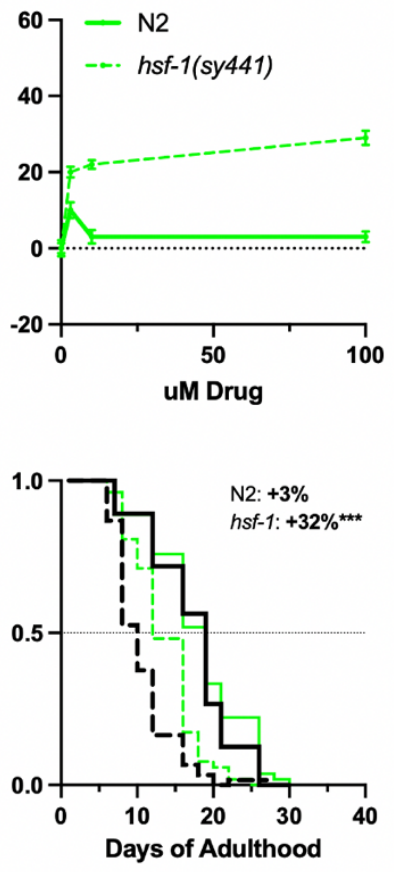

- N2 - DMSO

- N2 - Lycorine

-- hsf-1(sy441) - DMSO

--. hsf-1(sy441) - Lycorine

D

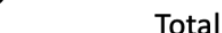

4E1RCat
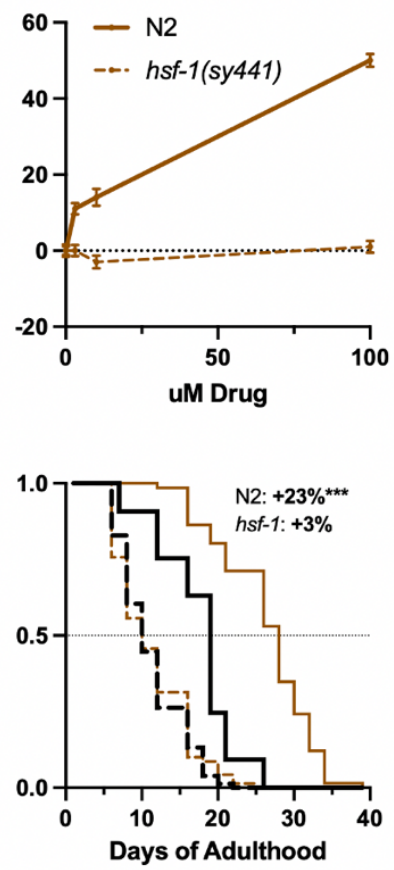

- N2 - DMSO

- N2 - 4E1RCat

-- hsf-1(sy441) - DMSO

--. hsf-1(sy441) - 4E1RCat
Total

Insoluble

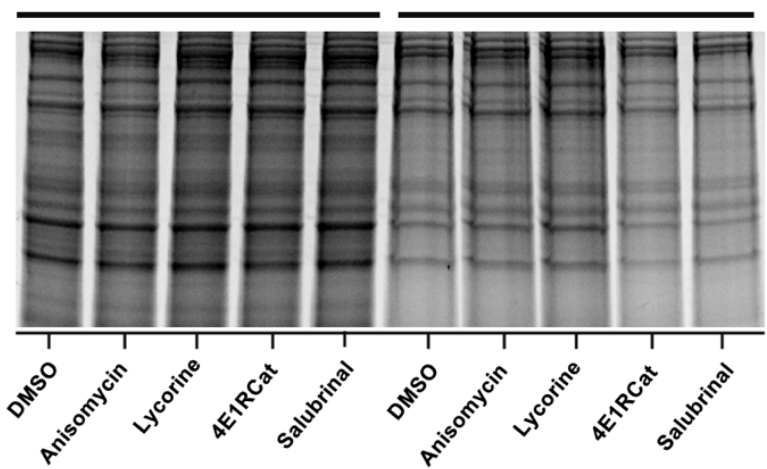

Salubrinal
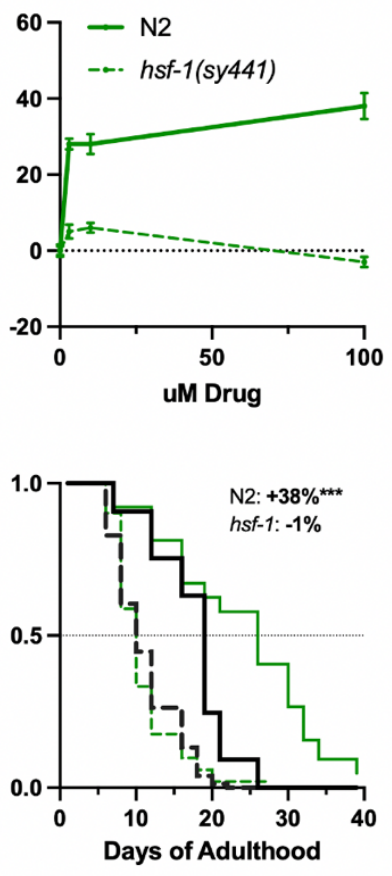

- N2 - DMSO

- N2 - Salubrinal

- $h s f-1(s y 441)$ - DMSO

--- hsf-1(sy441) - Salubrinal

E

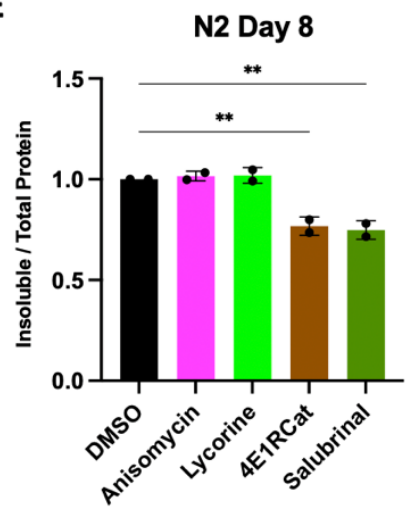

Figure 5: Reciprocal lifespan extension by translation inhibitors in hsf-1(sy441) animals

A) Mean lifespan as a function of translation inhibitor concentration. A maximum increase in lifespan of N2 animals (dashed black line) was seen at a $100 \mu \mathrm{M}$ concentration. The lifespan of $h s f-1$ (sy441) was tested in parallel, where the effects of initiation versus elongation inhibitors were reversed. Error bars indicate \pm SEM. See Supplementary Table 1 for details.

B) Survival curves from representative experiments show the fraction of wild-type (N2, solid line) or hsf-1-deficient (dashed line) animals when treated with $100 \mu \mathrm{M}$ of the indicated compound. Black lines indicate DMSO treatment, and colored lines indicate inhibitor treatment. Data are displayed as a Kaplan-Meier survival curve and significance determined by log-rank test. C) Experimental strategy for treating animals with inhibitors and isolating detergent-insoluble fractions. First, 10,000 animals were treated and allowed to age for 8 days before being washed with $M 9$, frozen in $N_{2}$, and mechanically lysed. Then proteins were extracted from the total lysate based on solubility, and an aliquot from each fraction was run on an SDS-PAGE gel.

D) Representative SDS-PAGE gel stained with the protein stain Sypro Ruby. 4E1RCat and salubrinal reduce the amount of ageassociated protein aggregation.

E) Quantification of 2 separate experiments shows 4E1RCAt and salubrinal to significantly reduce age-associated aggregation in wild-type (N2) animals. Data are displayed as mean \pm SEM and $* *=p<0.01$ by two-tailed students t-test. 
Lifespan extension by translational elongation and initiation inhibitors is dictated by genetic background

Lowering translation is an established mechanism to extend lifespan and delay aging (Anisimova et al., 2018; Klaips et al., 2018; Steffen and Dillin, 2016). Furthermore, aging is a well-known driver of protein aggregation. However, to our knowledge, it has never been investigated if the anti-aggregation and anti-aging effects of translational inhibition can be uncoupled and if the mode of translational inhibition influences these phenotypes. Inhibition of translation in wild-type animals, using the two elongation inhibitors anisomycin and lycorine, showed no, or only a minor lifespan extension in N2 animals (Fig 5A). In contrast, inhibition of translation by the initiation inhibitors 4E1RCat and salubrinal dose-dependently extended lifespan (Fig. 5B). This difference was observed despite that all four translation inhibitors reduced protein translation to the same extent (Fig. 1A). Thus, the difference in the effect on lifespan by elongation inhibitors and initiation inhibitors cannot be explained by reducing overall protein synthesis alone.

As the ability of the initiation inhibitors 4E1RCat and salubrinal to protect from heat stress was dependent on the transcription factor HSF-1, we asked if they were able to extend the lifespan of $h s f-1$ (sy441) mutants. Both 4E1RCat and salubrinal failed to significantly extend lifespan in $h s f-1$ (sy441) mutants. This result is consistent with the model put forward by Rodgers et al., which proposes that inhibition of translation initiation extends lifespan by lowering overall translation while selectively promoting the translation of cap-independent transcripts, many of which are transcribed by HSF-1.

However, to our surprise, treatment with the elongation inhibitors anisomycin and lycorine extended the lifespan of $h s f-1$ (sy441) mutants by $20 \%$ (Fig. 5B). We interpret this lifespan extension as a partial rescue of the protein folding defect in $h s f-1$ (sy441) mutants as the increase in lifespan did not reach the lifespan of wild-type animals. Taken together, however, our data demonstrate that longevity induced by translation inhibition subsumes several different mechanisms that lead to longevity.

We next tested the ability of all four inhibitors to reduce age-associated protein aggregation. If inhibition of translation alone is sufficient to reduce protein aggregation independently of any downstream mechanisms, all four inhibitors should reduce age-associated protein aggregation. Conversely, if the reduction of age-associated protein aggregation is closely linked to longevity, then only the initiation inhibitors should reduce protein aggregation. We treated day $1, \mathrm{~N} 2$ animals with $100 \mu \mathrm{M}$ of each of the four inhibitors and allowed the animals to age for eight days, after which we separated proteins based on solubility (Figure $5 \mathrm{C}$ ). We found that only the two initiation inhibitors that extended lifespan caused significant decreases in the amount of SDS-insoluble aggregates and that the elongation inhibitors failed to do so (Figure 5D \& E).

Taken together, these data support a model in which inhibition of translation elongation rescues proteostasiscompromised animals by reducing folding load but without generating additional protein folding capacity. Separately, inhibition of translation initiation protects and improves longevity through selective translation that activates an HSF-1 dependent mechanism. However, inhibition of translation initiation does not rescue or even can further exacerbate damage in animals with preexisting proteostasis imbalance. 


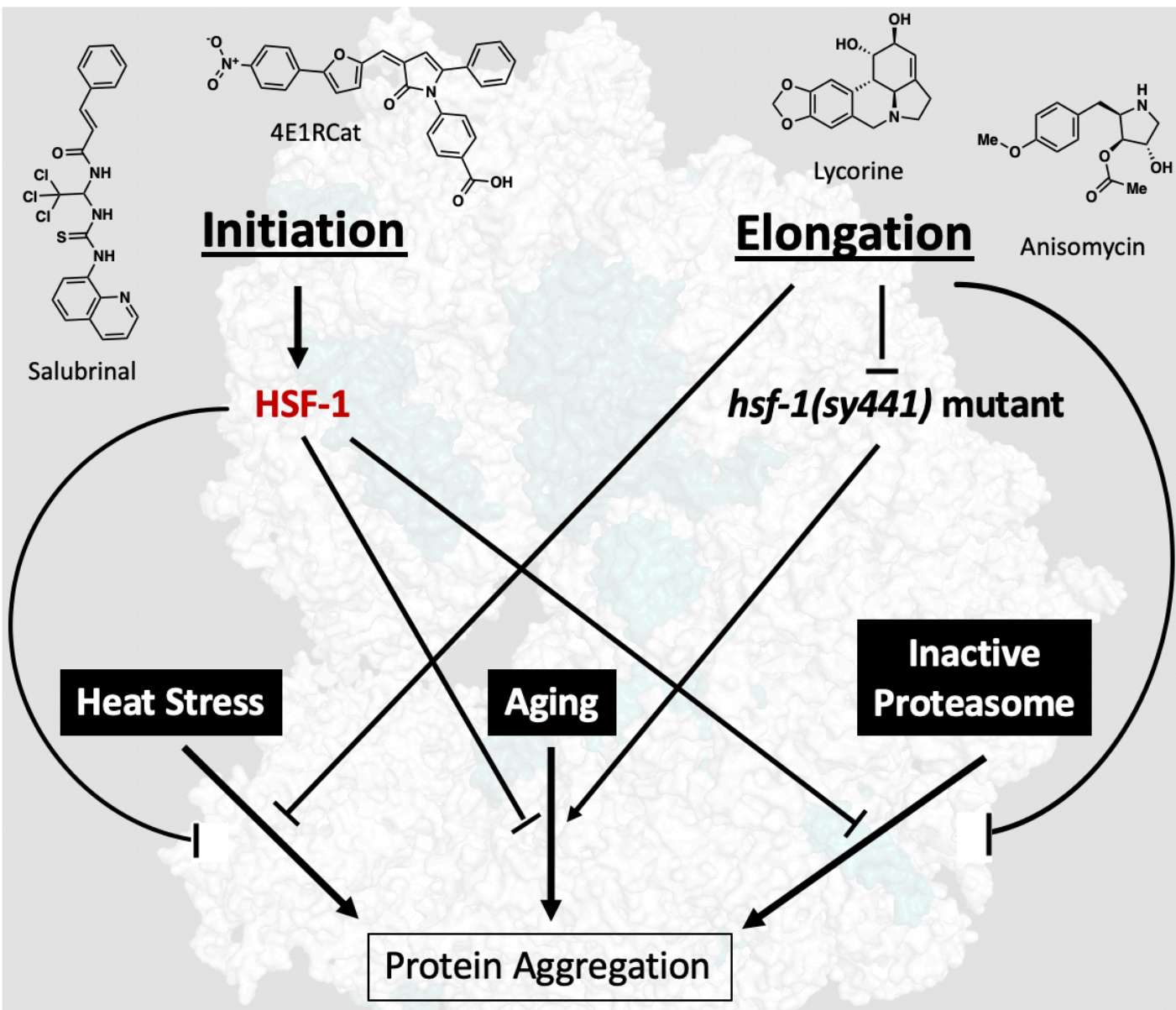

Figure 6: Consequences of proteotoxicity on aggregation at the ribosomal interface Initiation inhibitors, salubrinal, and 4E1RCat require $h s f-1$ to elicit a protective response following proteotoxic stress, functioning similar, if not overlapping with, canonical stress responses. Conversely, elongation inhibitors, anisomycin, and lycorine do not boost protection in healthy wild-type animals but rescue proteotoxicity in animals with preexisting proteostasis deficits such as hsf-1 mutants or animals expression misfolded proteins.

\section{Discussion}

In this study, we set out to answer how translation inhibition exerts its beneficial effects on proteostasis and longevity in the metazoan C. elegans. We considered two previously proposed models (Medicherla and Goldberg, 2008; Rogers et al., 2011; Seo et al., 2013; Zhou et al., 2014). The selective translation model proposes that inhibition of translation improves proteostasis by an active mechanism remodeling the proteome through differential translation. In the selective translation model, the system increases folding capacity through the increased translation of HSF-1 targets and by proteasomal degradation of preexisting proteins. The second model, the reduced folding load model, proposes that inhibition of translation improves proteostasis by lowering the concentration of newly synthesized proteins, thereby increasing relative folding capacity via a reduced folding load on the proteostasis machinery.

Our data establish evidence for both models. We show that the mode of translation inhibition determines the mechanism of proteome protection. Translation initiation inhibitors initiate a selective translation mechanism 
that requires HSF-1 and the proteasome (Howard et al., 2016; Rogers et al., 2011; Seo et al., 2013). In contrast, elongation inhibitors reduce folding load and do not require HSF-1 or the proteasome. Elongation inhibitors rescue deficiencies induced by the lack of these components. What was particularly striking to us was how cleanly different modes of translational inhibition could be separated based on the stressor. The most general conclusion that we draw from our study is that inhibition of translation can trigger genetically and biochemically distinct, HSF-1 dependent and independent proteostasis mechanisms.

Translation can be separated into the three major steps, initiation, elongation, and termination, each of which can be further subdivided into several minor steps. Translation is further regulated by signaling factors and ribosome assembly rates, which are also subject to proteostatic intervention. Our data show that the phenotypic consequences of lowering translation depend on the environmental context and the inhibited step of translation. It remains to be seen if modulation of additional steps, such as termination, triggers additional genetically distinct proteostasis mechanisms.

Inhibiting either initiation or elongation protects from the damaging effects of heat shock. Otherwise, the two protective mechanisms are complimentary, with one being protective while the other is not. Overall, our data suggest that initiation inhibitors remodel the proteome in an HSF-1 and proteasome-dependent manner, as previously suggested. In contrast, elongation inhibitors act independently of both HSF-1 and the proteasome, thereby rescuing the phenotypic consequences of their inactivation. The most striking example of this dichotomy was seen when preexisting folding problems-caused by the lack of either HSF-1 or the expression of the aggregation-prone polyQ protein -were exacerbated by the chemical inhibition of the proteasome. These combined insults led to increased protein aggregation, uncoordination, shrinking body size, and death of the animals. Inhibition of elongation almost entirely rescued these phenotypes. In sharp contrast, inhibition of initiation made the animals significantly worse and increased death. Our data reveal that the mode by which translation is inhibited leads to the activation of distinct downstream mechanisms that protect the organism from different insults.

A second striking example observed was the ability of initiation inhibitors to extend lifespan while the elongation inhibitors did not. A long-standing question in the field of longevity is if lowering protein synthesis by inhibiting translation is sufficient to extend lifespan. Alternative explanations for the observed longevity include lowering the expression of specific proteins (e.g., DAF-2) or the activation of a specific downstream mechanism that is activated by low translation rates (Howard et al., 2016). Our data suggest some proteome remodeling may be necessary to extend lifespan (Koyuncu et al., 2021).

The lack of longevity from anisomycin and lycorine treatment in our experiments is unlikely to be the result of toxicity due to an off-target effect. First, lycorine and anisomycin are structurally very distinct and are unlikely to share off-targets. Second, and more importantly, both extend the lifespan of $h s f-1(s y 441)$ mutants, which strongly argues against a toxic side effect. In general, we observed that elongation inhibitors are good at rescuing defects in strains with impaired proteostasis. In strains with impaired proteostasis, inhibition of elongation lowers the production of newly synthesized proteins and thus reduces the folding load on the proteostasis machinery. This effect alleviates folding problems and thus allows the elongation inhibitors to rescue the shortened lifespan of proteostasis-deficient strains. 
Ideas and Speculation

The ability of elongation inhibitors to improve proteostasis in strains with preexisting folding defects on the organismal level raises the interesting question of whether they could be developed therapeutically. It has often been suggested that the protein degradation machinery such as autophagy or the proteasome is overloaded in protein folding diseases. If the $C$. elegans results translate to mammals, treating a mouse model of a proteinfolding disease with an initiation inhibitor would be detrimental. In contrast, treatment with an elongation inhibitor would be beneficial. Furthermore, these $C$. elegans findings may explain some surprising previous observations. For example, treating the SOD1 G93A mouse model of amyotrophic lateral sclerosis (ALS) with rapamycin, a drug modulating translational initiation through the phosphorylation of $4 \mathrm{E}-\mathrm{BP} 1$ exacerbates ALS disease phenotypes (Zhang et al., 2011). If the logic we uncovered for C. elegans applies to the SOD1 G93A model, an elongation inhibitor given at an intermediate concentration to lower protein synthesis by $30 \%$ should rescue some of the disease phenotypes.

Proposing translation inhibitors as potential therapeutics first seems counterintuitive and dangerous. However, besides rapamycin, emetine and homoharringtonine are two additional FDA-approved elongation inhibitors. Emetine is a eukaryote-specific translation inhibitor with a long history of being used in humans, but its nauseainducing properties make chronic use unsustainable. Homoharringtonine is an elongation inhibitor that only binds vacant ribosomes that can no longer access its binding site once translation has commenced. Essentially it functions as an initiation inhibitor that binds within the ribosomal peptidyl transferase center (Dmitriev et al., 2020). Finally, the tetracyclines, minocycline, and doxycycline, also act as translation inhibitors for both mitochondrial translation and cytoplasmic translation (Molenaars et al., 2020; Mortison et al., 2018; Solis et al., 2018). These examples show that, as long as cytoplasmic translation is reduced rather than wholly blocked, translation inhibitors are tolerable in humans. Thus, translation inhibitors may be suitable for therapeutic caused by the disease. 
bioRxiv preprint doi: https://doi.org/10.1101/2022.01.23.477418; this version posted January 24,2022 . The copyright holder has placed this preprint (which was not certified by peer review) in the Public Domain. It is no longer restricted by copyright. Anyone can legally share, reuse, remix, or adapt this material for any purpose without crediting the original authors.

\section{S1}

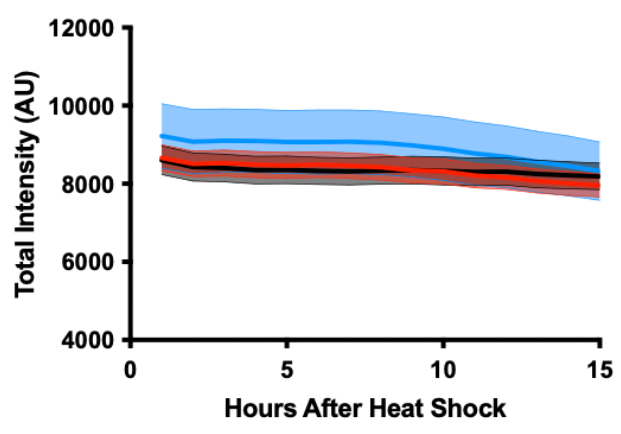

- NoHS $n=31$

- $1 \mathrm{Hr} \quad \mathrm{n}=35$

$-2 \mathrm{Hr} \quad \mathrm{n}=27$

54

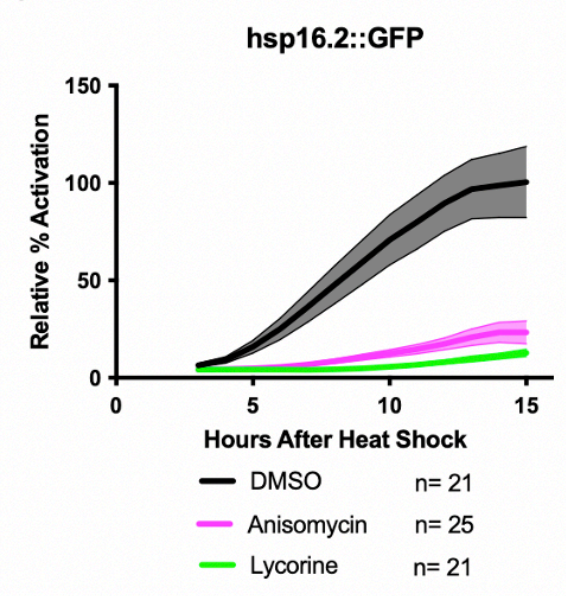

S2

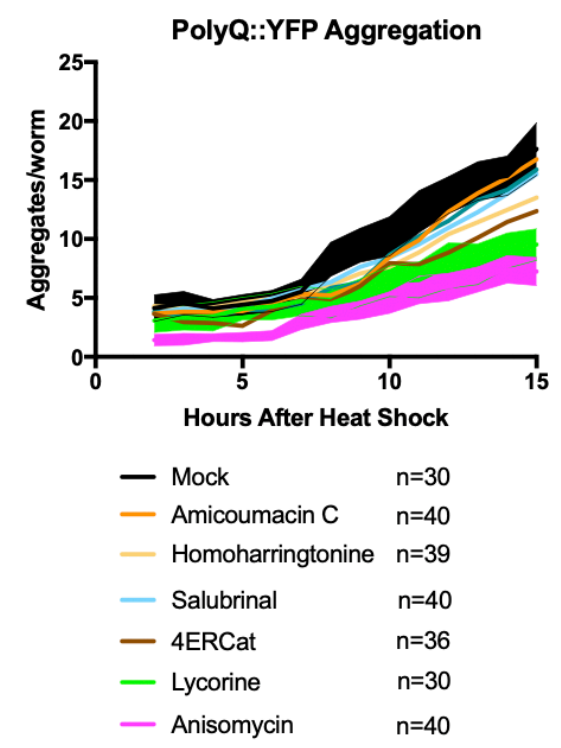

S3

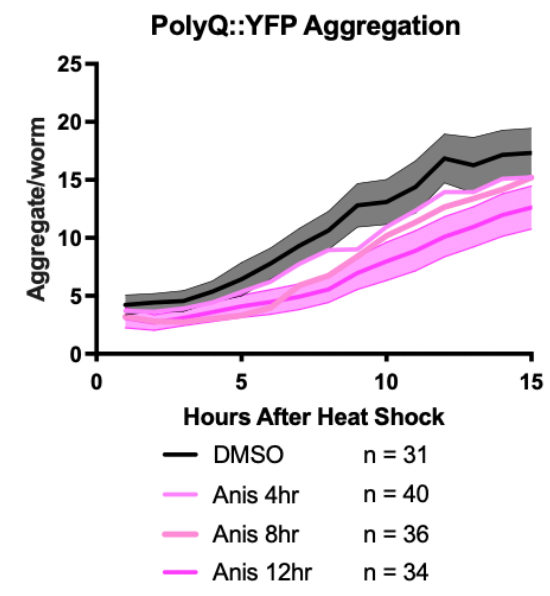

S5<smiles>COc1ccc(CC2NC[C@@H](O)C2OC(C)=O)cc1</smiles>

Anisomycin<smiles></smiles>

Lycorine<smiles>O=C(/C=C/c1ccccc1)NC(NC(=S)Nc1cccc2cccnc12)C(Cl)(Cl)Cl</smiles>

4E1RCat

\section{Supplemental Figure:}

S1) Total fluorescent YFP intensity in PolyQ transgenic animals does not change significantly within the 15 hours imaging of the animals after the HS, showing that the aggregate formation is a redistribution of soluble PolyQ::YFP into aggregation foci. Lines indicate mean, and shading indicates $95 \% \mathrm{Cl}$.

S2) Transgenic AM140 worms treated with indicated chemicals for 12 hours at the late L4 stage, then subjected to $36^{\circ} \mathrm{C}$ for 2 hours on day 1 of adulthood. Only anisomycin and lycorine had suppressed aggregate formation as measured in our live imaging protocol. Lines indicate mean, and shading indicates $95 \% \mathrm{Cl}$ for DMSO, anisomycin, and lycorine.

S3) 12-hour pre-treatment of anisomycin in PolyQ C. elegans was necessary to inhibit aggregation significantly. Lines indicate means, while DMSO and 12-hour treatment with anisomycin $(100 \mu \mathrm{M})$ include $95 \% \mathrm{Cl}$ as indicated by shading.

S4) Elongation inhibitors suppressed the HSR as measured by $h s p-16.2::$ GFP fluorescence assay. After incubation with the inhibitors for 4 days followed by a 1 hour HS at $36^{\circ} \mathrm{C}$, little to no increase in GFP expression was observed, indicating that all inhibitors block HSR activation at the tested concentration $(100 \mu \mathrm{M})$. Lines indicate mean, and shading indicates $95 \% \mathrm{Cl}$. S5) Chemical structures of anisomycin, lycorine, 4E1RCat, and salubrinal. 
bioRxiv preprint doi: https://doi.org/10.1101/2022.01.23.477418; this version posted January 24, 2022. The copyright holder has placed this

preprint (which was not certified by peer review) in the Public Domain. It is no longer restricted by copyright. Anyone can legally share, reuse, remix, or adapt this material for any purpose without crediting the original authors.

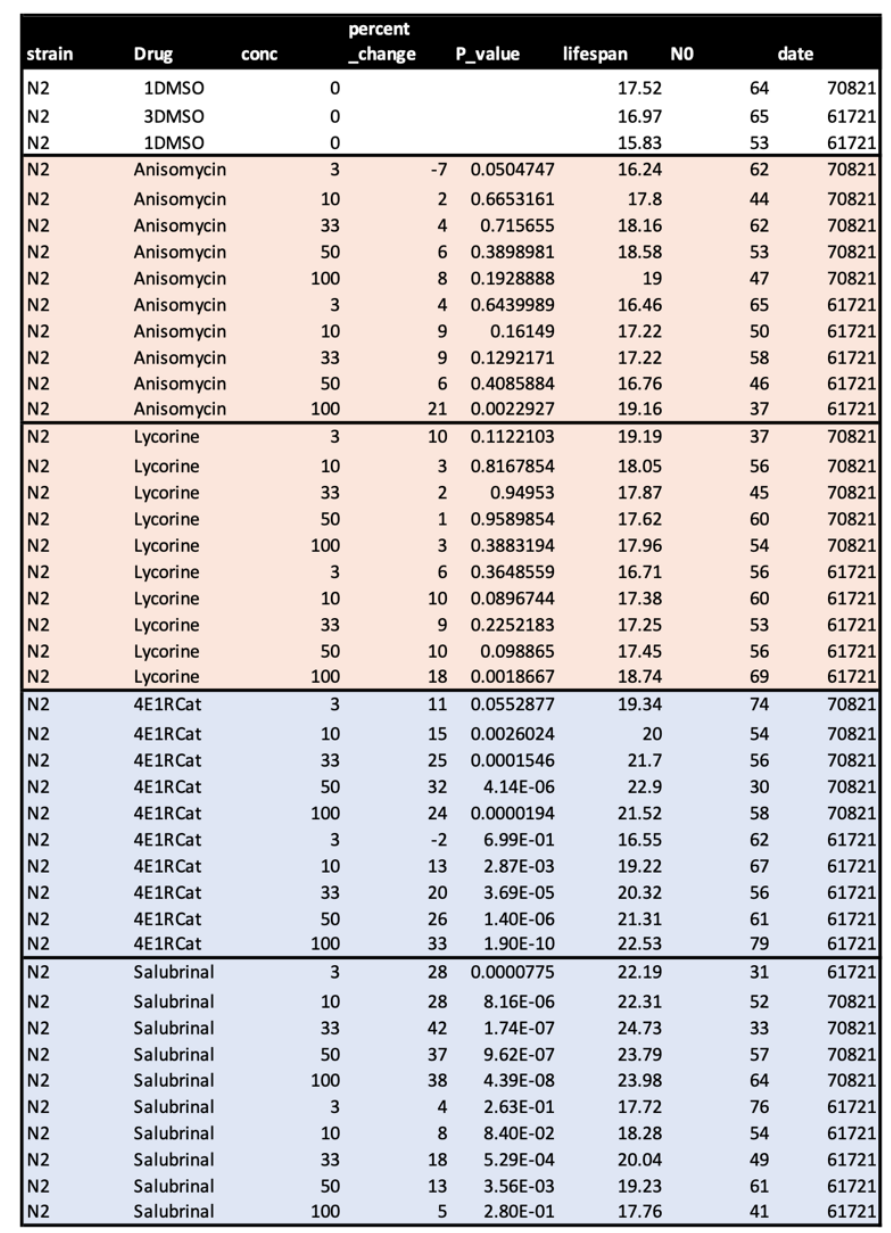

Supplemental Table 1:

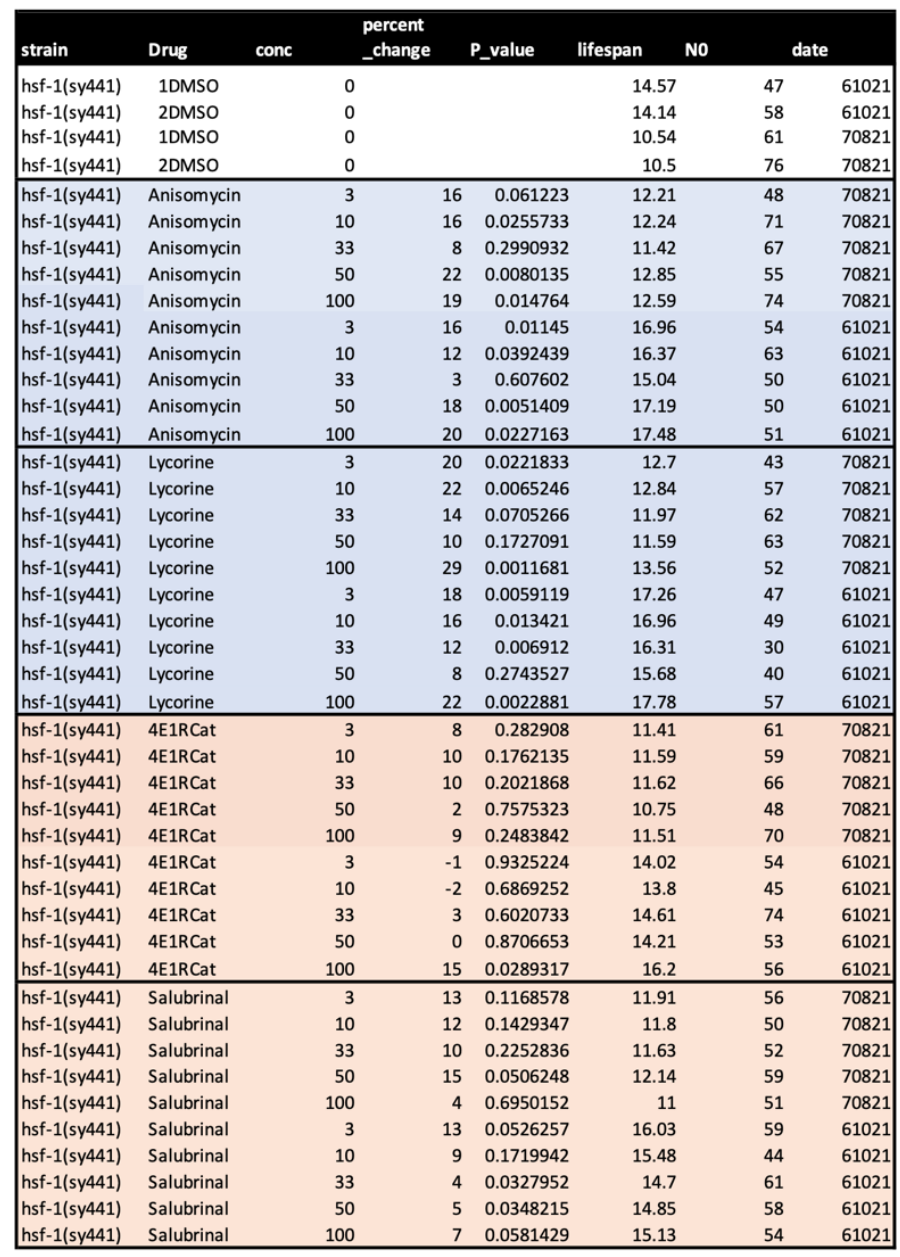

Representative dose-response curves of DMSO, anisomycin, lycorine, 4E1RCat, and salubrinal in 96-well plate lifespan assays, repeated in two complete individual replicates. For N2, only 4ER1RCat and salubrinal robustly increase lifespan (blue), while anisomycin and lycorine either are not effective or slightly toxic (red). For $h s f-1(s y 441)$, only anisomycin and lycorine robustly increase lifespan (blue), while 4E1RCat and salubrinal either are not effective or slightly toxic (red). 


\section{Acknowledgments}

We would like to acknowledge Drs Anabel Perez-Gomez, Sarah Ly, Jin Lee, Caroline Kumsta, and Malene Hansen for input into the manuscript; Alan To for technical assistance; the Yusupov lab for generating the structures of anisomycin, lycorine, and homoharringtonine in complex with the eukaryotic ribosome; and Dr. Shigefumi Kuwahara (Tohoku University) for providing Amicoumacin C. Grants supported this work to M.P. from the NIH (DP2 OD008398, R21NS107951, R01AG067331), and the Glenn Foundation. K.C. was funded by the Dorris Neuroscience Scholar Fellowship. Some strains were provided by the CGC, which is funded by the NIH Office of Research Infrastructure Programs (P40 OD010440).

Competing interests: M.P. and K.C are scientific founders and advisors to Cyclone Therapeutics, Inc., a biotech company developing therapeutics targeting translation.

\section{$\underline{\text { Lead Contact and Materials Availability }}$}

Michael Petrascheck is the Lead Contact and may be contacted at pscheck@scripps.edu. This study did not generate new unique reagents; however, the natural product Amicoumacin $\mathrm{C}$ was obtained as a gift from Dr. Shigefumi Kuwahara, Ph.D. (Tohoku University). This reagent is not available without total chemical synthesis.

\section{Experimental Model and Subject Details}

\section{C. elegans Strains}

The Bristol strain (N2) was used as the wild-type strain. The following worm strains used in this study were obtained from the Caenorhabditis Genetics Center (CGC; Minneapolis, MN): CL2070 [dvls70 [hsp-16.2p::GFP + rol-6(su1006)]], AM140 [rmls132[Punc-54::q35::yfp]] and PS3551 [hsf-1(sy441)].

\section{$\underline{\text { Method Details }}$}

\section{Worm Maintenance}

1000 - 2000 age-synchronized animals were plated into $6 \mathrm{~cm}$ culture plates with liquid medium (S-complete medium with $50 \mathrm{mg} / \mathrm{mL}$ carbenicillin and $0.1 \mathrm{mg} / \mathrm{mL}$ fungizone (Amphotericin B)) containing $6 \mathrm{mg} / \mathrm{mL} \mathrm{X-ray}$ irradiated Escherichia coli OP50 (1.5 x $10^{8}$ colony-forming units [cfu]/ml, carbenicillin-resistant to exclude growth of other bacteria), freshly prepared 4 days in advance, as previously described (Solis and Petrascheck, 2011), and were maintained at $20^{\circ} \mathrm{C}$. The final volume in each plate was $7 \mathrm{~mL}$. To prevent self-fertilization, FUDR (5-fluoro-2'-deoxyuridine, 0.12 mM final) (Sigma- Aldrich, cat \# 856657) was added 42-45 hours after seeding. At the late $\mathrm{L} 4$ stage, either DMSO/drug treatment (100 $\mu \mathrm{M}$ unless otherwise stated) was added to each strain.

\section{Surface sensing of translation (SUnSET) to analyze the effectiveness of translation inhibitors in C. elegans}

Day 1 adult N2 worms were bleached, and eggs were allowed to hatch in S-complete by shaking them overnight. On the next day, 12,000 L1 worms were seeded in a $15 \mathrm{~cm}$ plate containing a total volume of $30 \mathrm{~mL}$ S-complete with $6 \mathrm{mg} / \mathrm{mL}$ OP50 bacteria, $50 \mu \mathrm{g} / \mathrm{mL}$ Carbenicillin, and $0.1 \mu \mathrm{g} / \mathrm{mL}$ Amphotericin B. $6 \mathrm{~mL}$ of $0.6 \mathrm{mM}$ Fluorodeoxyuridine (FUDR) were added to worms at L4 stage in each plate. $100 \mu \mathrm{M}$ translation inhibitor was added to worms 2 hours after adding FUDR. After 12 hours, worms were transferred into a $15 \mathrm{~mL}$ corning tube containing a total volume of $5 \mathrm{~mL} \mathrm{~S}$-complete with $750 \mathrm{ul} 6 \mathrm{mg} / \mathrm{mL}$ OP50 bacteria, $0.5 \mathrm{mg} / \mathrm{mL}$ puromycin, and 
$100 \mu \mathrm{M}$ translation inhibitors. After rotating the corning tubes for $4 \mathrm{~h}$, worms were collected into $2 \mathrm{~mL}$ cryotubes by washing them with M9 once and with cold PBS 3 times. Worms were flash-frozen in liquid nitrogen and subsequently broken with a beak mill homogenizer (Fisherbrand). Protein concentrations were determined by the Bradford protein assay. $50 \mathrm{mg}$ protein from each sample was loaded for western blot analysis using antibodies against puromycin (Millipore, MABE343) and GAPDH (Proteintech, 10494-1-AP). Antibodies were diluted 1:5,000 in 5\% non-fat milk in TBST.

\section{Thermotolerance}

Age-synchronized N2 or PS3551 [hsf-1(sy441)] animals were prepared as above in $6 \mathrm{~cm}$ culture plates and treated with water or $100 \mathrm{mM}$ lycorine/anisomycin on day 1. On day 4, 25-35 animals were transferred to 6 $\mathrm{cm}$ NGM plates in triplicate for each condition and were transferred to the non-permissive temperature of 36 ${ }^{\circ} \mathrm{C}$. Every hour, survival was scored by lightly touching animals with a worm pick and scoring for movement.

\section{C. elegans insoluble protein extraction}

10,000 N2 worms were sorted into a $15 \mathrm{~cm}$ liquid culture dish using the COPAS Biosorter. For heat shock-induced aggregation experiments, worms were treated with either DMSO or anisomycin $(100 \mu \mathrm{M})$ for 12 hours on Day 1 of adulthood, then subjected to a 2-hour heat shock at $36^{\circ} \mathrm{C}$. After 12 hours of recovery, the animals were washed 3 times with S-complete buffer, once with PBS, and then flash-frozen in liquid nitrogen. 500uL of cold lysis buffer $(20 \mathrm{mM}$ Tris base, $100 \mathrm{mM} \mathrm{NaCl}, 1 \mathrm{mM} \mathrm{MgCl}$, $\mathrm{pH}=7.4$, with protease inhibitors (Roche, 11836153001) was added, and animals homogenized mechanically. An aliquot of this total lysate was saved. In an ultracentrifuge tube, 2 volumes of SDS Extraction buffer $(20 \mathrm{mM}$ Tris base, $100 \mathrm{mM} \mathrm{NaCl}, 1 \mathrm{mM} \mathrm{MgCl} 2, \mathrm{pH}=$ 7.4 , with protease inhibitors, and $1 \%$ SDS) was added to 1 volume of total lysate and was centrifuged at 20,000 $x \mathrm{~g}$ for 30 minutes. The extraction was repeated 2 times to remove all SDS soluble proteins. The remaining insoluble pellet was suspended in $20 \mathrm{uL}$ urea buffer ( $8 \mathrm{M}$ urea, 50mM DTT, $2 \%$ SDS, 20mM Tris base, $\mathrm{pH}=7.4$ ) and sonicated briefly. 18uL of the insoluble suspension was added to 6uL 4x Laemmli buffer (Biorad, \#161-0747) supplemented with 10\% 2-mercaptoethanol (Sigma, 60-24-2) and boiled for 5 minutes, then directly loaded onto SDS-Page gel (Biorad, 4569033). Gels were stained with Sypro Ruby according to the manufactures directions and quantified in ImageJ.

For age-associated protein aggregation experiments, the above was repeated with the following changes: Day 1 worms were treated with $100 \mu \mathrm{M}$ of each compound and allowed to age in liquid culture until Day 8 of adulthood. Following lysis, worms were washed several times with PBS to remove soluble protein.

\section{Proteasome dysfunction assay-Survival}

Animals were prepared as above in 96 well plates. At the late L4 stage, animals were pretreated with DMSO or $100 \mu \mathrm{M}$ anisomycin. After 12 hours, the animals were treated with $75 \mu \mathrm{M}$ bortezomib. On day 8 of adulthood, the percent of animals alive was determined by movement in liquid culture.

\section{Proteasome dysfunction assay-Worm Length}

Animals were prepared as above in $6 \mathrm{~cm}$ liquid culture dishes. At the late L4 stage, animals were pretreated with DMSO or $100 \mu \mathrm{M}$ anisomycin. After 12 hours, the animals were treated with $75 \mu \mathrm{M}$ bortezomib. Body length was measured on day 3 for $h s f-1$ (sy441) or day 5 for PolyQ using the 10x objective with the ImageXpress Micro $\mathrm{XL}$ and Metaexpress microscopy software. 
Heat Shock Induced Aggregation and Stress Response

CL2070 [dvls70 [hsp-16.2p::GFP + rol-6(su1006)]] or AM140 [rmls132[Punc-54::q35::yfp]] age-synchronized animals were treated with DMSO or $100 \mu \mathrm{M}$ drug at late L4. 4-12 hours later, on day 1 of adulthood, $1.5 \mathrm{~mL}$ of the treated animals were transferred from liquid culture into an Eppendorf tube, washed twice with S-complete, pelleted, then transferred to $6 \mathrm{~cm}$ NGM plates using S-complete. Once the animals were completely dry on the NGM plate, they were transferred to a $36^{\circ} \mathrm{C}$ incubator, plates upside down, for $1-2$ hours.

\section{Hydrogel Mounting}

Animals were washed from NGM plates using 0.2\% HHPPA (2-hydroxy-4'-(2-hydroxyethoxy)-2methylpropiophenone) (CAS[106797-53-9]) dissolved in S-complete, into a $2 \mathrm{~mL}$ Eppendorf tube. Animals were washed twice with $0.2 \%$ HHPPA, then suspended in $0.3 \mathrm{~mL} 0.2 \%$ HHPPA. $2.5 \mathrm{uL}$ of this solution was seeded into a single well of a 384-well plate containing 2.5 uL 30\% PEG-DA (polyethylene glycol diacrylate, $M W=4000$, Polysciences, cat \# 15246-1) in S-complete. After 5 minutes to allow the solutions to diffuse together, the animals were immobilized by subjecting the 384-well plate UV light using a routine laboratory gel viewer (UVP Dual-Intensity Ultraviolet Transilluminator, high intensity) for 30 seconds. 45 uL S-complete buffer was added on top to prevent desiccation. In general, each well contained 5-10 worms.

\section{Imaging and Analysis}

Time-lapse brightfield and fluorescence images were taken with a 10x objective using the ImageXpress Micro XL over 15 hours. The number of PolyQ aggregates, or total YFP fluorescence, in the whole worm was determined by analyzing images using a custom pipeline created in CellProfiler.

\section{Lifespan Assay}

Age-synchronized C. elegans were prepared in liquid medium, as described above, and seeded into flat-bottom, optically clear 96-well plates (Corning, 351172) containing 150uL total volume per well, as previously described (Clay and Petrascheck, 2020). Plates contained $\sim 10$ animals per well in $6 \mathrm{mg} / \mathrm{mL} \gamma$-irradiated OP50. Agesynchronized animals were seeded as $\mathrm{L} 1$ larvae and grown at $20^{\circ} \mathrm{C}$. Plates were covered with sealers to prevent evaporation. To prevent self-fertilization, FUDR $(0.12 \mathrm{mM}$ final) was added $42-45$ hours after seeding. Drugs were added on day 1 of adulthood. When used, DMSO was kept to a final concentration of $0.33 \% \mathrm{v} / \mathrm{v}$.

\section{Quantification and Statistical Analysis}

\section{Aggregation and Induction of the HSR}

The number of $\mathrm{n}$ represents the total number of animals over three individual experiments. For the pairedtime-lapse data generated, we chose to depict the $95 \%$ confidence interval, which was calculated using Graphpad Prism, to show differences in treatment.

\section{Percent Survival - Thermotolerance assay}

The number of $\mathrm{n}$ represents the total number of animals over the 3 individual replicates shown as the average percentage survival and SEM, calculated using Graphpad Prism. Significance was determined by using a rowmatched two-way ANOVA with Šídák multiple comparisons test. 
Worm Length - Proteasome dysfunction assay

The number of $\mathrm{n}$ represents the total number of animals whose length was measured in one experiment. Depicted are the mean and standard deviation calculated using Graphpad Prism. Significance was determined by the two-tail unpaired t-test. Similar results were observed across 3 independent experiments.

\section{Lifespan Assay}

Survival was scored manually by visually monitoring worm movement using an inverted microscope 3 times per week. Statistical analysis was performed using the Mantel-Haenzel version of the log-rank test as outlined in Petrascheck and Miller (Petrascheck and Miller, 2017).

\section{Data and Code Availability}

The software used in this study (Cell Profiler) is available at cellprofiler.org

\section{References}

Anisimova, A.S., Alexandrov, A.I., Makarova, N.E., Gladyshev, V.N., and Dmitriev, S.E. (2018). Protein synthesis and quality control in aging. Aging 10, 4269-4288.

Arnold, A., Rahman, M.M., Lee, M.C., Muehlhaeusser, S., Katic, I., Gaidatzis, D., Hess, D., Scheckel, C., Wright, J.E., Stetak, A., et al. (2014). Functional characterization of C. elegans Y-box-binding proteins reveals tissuespecific functions and a critical role in the formation of polysomes. Nucleic acids research 42, 13353-13369.

Balch, W.E., Morimoto, R.I., Dillin, A., and Kelly, J.W. (2008). Adapting proteostasis for disease intervention. Science (New York, NY) 319, 916-919.

Brignull, H.R., Morley, J.F., Garcia, S.M., and Morimoto, R.I. (2006). Modeling polyglutamine pathogenesis in C. elegans. Methods Enzymol 412, 256-282.

Choe, Y.J., Park, S.H., Hassemer, T., Korner, R., Vincenz-Donnelly, L., Hayer-Hartl, M., and Hartl, F.U. (2016). Failure of RQC machinery causes protein aggregation and proteotoxic stress. Nature 531, 191-195.

Chu, J., Hong, N.A., Masuda, C.A., Jenkins, B.V., Nelms, K.A., Goodnow, C.C., Glynne, R.J., Wu, H., Masliah, E., Joazeiro, C.A., et al. (2009). A mouse forward genetics screen identifies LISTERIN as an E3 ubiquitin ligase involved in neurodegeneration. Proc Natl Acad Sci U S A 106, 2097-2103.

Clay, K.J., and Petrascheck, M. (2020). Design and Analysis of Pharmacological Studies in Aging. In Aging: Methods and Protocols, S.P. Curran, ed. (New York, NY: Springer US), pp. 77-89.

David, D.C., Ollikainen, N., Trinidad, J.C., Cary, M.P., Burlingame, A.L., and Kenyon, C. (2010). Widespread Protein Aggregation as an Inherent Part of Aging in C. elegans. PLOS Biology 8, e1000450.

Dmitriev, S.E., Vladimirov, D.O., and Lashkevich, K.A. (2020). A Quick Guide to Small-Molecule Inhibitors of Eukaryotic Protein Synthesis. Biochemistry (Mosc) 85, 1389-1421. 
Garreau de Loubresse, N., Prokhorova, I., Holtkamp, W., Rodnina, M.V., Yusupova, G., and Yusupov, M. (2014). Structural basis for the inhibition of the eukaryotic ribosome. Nature 513, 517-522.

Higuchi-Sanabria, R., Frankino, P.A., Paul, J.W., 3rd, Tronnes, S.U., and Dillin, A. (2018). A Futile Battle? Protein Quality Control and the Stress of Aging. Developmental cell 44, 139-163.

Howard, A.C., Rollins, J., Snow, S., Castor, S., and Rogers, A.N. (2016). Reducing translation through elF4G/IFG1 improves survival under ER stress that depends on heat shock factor HSF-1 in Caenorhabditis elegans. Aging cell 15, 1027-1038.

Klaips, C.L., Jayaraj, G.G., and Hartl, F.U. (2018). Pathways of cellular proteostasis in aging and disease. The Journal of Cell Biology 217, 51-63.

Koyuncu, S., Loureiro, R., Lee, H.J., Wagle, P., Krueger, M., and Vilchez, D. (2021). Rewiring of the ubiquitinated proteome determines ageing in C. elegans. Nature 596, 285-290.

Lan, J., Rollins, J.A., Zang, X., Wu, D., Zou, L., Wang, Z., Ye, C., Wu, Z., Kapahi, P., Rogers, A.N., et al. (2019). Translational Regulation of Non-autonomous Mitochondrial Stress Response Promotes Longevity. Cell Reports 28, 1050-1062.e1056.

McQuary, P.R., Liao, C.Y., Chang, J.T., Kumsta, C., She, X., Davis, A., Chu, C.C., Gelino, S., Gomez-Amaro, R.L., Petrascheck, M., et al. (2016). C. elegans S6K Mutants Require a Creatine-Kinase-like Effector for Lifespan Extension. Cell Rep 14, 2059-2067.

Medicherla, B., and Goldberg, A.L. (2008). Heat shock and oxygen radicals stimulate ubiquitin-dependent degradation mainly of newly synthesized proteins. J Cell Biol 182, 663-673.

Molenaars, M., Janssens, G.E., Williams, E.G., Jongejan, A., Lan, J., Rabot, S., Joly, F., Moerland, P.D., Schomakers, B.V., Lezzerini, M., et al. (2020). A Conserved Mito-Cytosolic Translational Balance Links Two Longevity Pathways. Cell Metabolism.

Morley, J.F., Brignull, H.R., Weyers, J.J., and Morimoto, R.I. (2002). The threshold for polyglutamine-expansion protein aggregation and cellular toxicity is dynamic and influenced by aging in Caenorhabditis elegans. Proc Natl Acad Sci U S A 99, 10417-10422.

Moronetti Mazzeo, L.E., Dersh, D., Boccitto, M., Kalb, R.G., and Lamitina, T. (2012). Stress and aging induce distinct polyQ protein aggregation states. Proc Natl Acad Sci U S A 109, 10587-10592.

Mortison, J.D., Schenone, M., Myers, J.A., Zhang, Z., Chen, L., Ciarlo, C., Comer, E., Natchiar, S.K., Carr, S.A., Klaholz, B.P., et al. (2018). Tetracyclines Modify Translation by Targeting Key Human rRNA Substructures. Cell Chem Biol 25, 1506-1518 e1513.

Nedialkova, Danny D., and Leidel, Sebastian A. (2015). Optimization of Codon Translation Rates via tRNA Modifications Maintains Proteome Integrity. Cell 161, 1606-1618.

Nollen, E.A., Garcia, S.M., van Haaften, G., Kim, S., Chavez, A., Morimoto, R.I., and Plasterk, R.H. (2004). Genomewide RNA interference screen identifies previously undescribed regulators of polyglutamine aggregation. Proc Natl Acad Sci U S A 101, 6403-6408. 
Petrascheck, M., and Miller, D.L. (2017). Computational Analysis of Lifespan Experiment Reproducibility. Front Genet 8, 92.

Reis-Rodrigues, P., Czerwieniec, G., Peters, T.W., Evani, U.S., Alavez, S., Gaman, E.A., Vantipalli, M., Mooney, S.D., Gibson, B.W., Lithgow, G.J., et al. (2012). Proteomic analysis of age-dependent changes in protein solubility identifies genes that modulate lifespan. Aging cell 11, 120-127.

Riback, J.A., Katanski, C.D., Kear-Scott, J.L., Pilipenko, E.V., Rojek, A.E., Sosnick, T.R., and Drummond, D.A. (2017). Stress-Triggered Phase Separation Is an Adaptive, Evolutionarily Tuned Response. Cell 168, 1028-1040.e1019.

Rogers, A.N., Chen, D., McColl, G., Czerwieniec, G., Felkey, K., Gibson, B.W., Hubbard, A., Melov, S., Lithgow, G.J., and Kapahi, P. (2011). Life span extension via elF4G inhibition is mediated by posttranscriptional remodeling of stress response gene expression in C. elegans. Cell Metab 14, 55-66.

Schrader, J., Henneberg, F., Mata, R.A., Tittmann, K., Schneider, T.R., Stark, H., Bourenkov, G., and Chari, A. (2016). The inhibition mechanism of human $20 \mathrm{~S}$ proteasomes enables next-generation inhibitor design. Science (New York, NY) 353, 594-598.

Seo, K., Choi, E., Lee, D., Jeong, D.-E., Jang, S.K., and Lee, S.-J. (2013). Heat shock factor 1 mediates the longevity conferred by inhibition of TOR and insulin/IGF-1 signaling pathways in C. elegans. Aging cell 12, 1073-1081.

Simonsen, A., Cumming, R.C., Brech, A., Isakson, P., Schubert, D.R., and Finley, K.D. (2008). Promoting basal levels of autophagy in the nervous system enhances longevity and oxidant resistance in adult Drosophila. Autophagy 4, 176-184.

Solis, G.M., Kardakaris, R., Valentine, E.R., Bar-Peled, L., Chen, A.L., Blewett, M.M., McCormick, M.A., Williamson, J.R., Kennedy, B., Cravatt, B.F., et al. (2018). Translation attenuation by minocycline enhances longevity and proteostasis in old post-stress-responsive organisms. Elife 7.

Steffen, K.K., and Dillin, A. (2016). A Ribosomal Perspective on Proteostasis and Aging. Cell Metab 23, 10041012.

Tye, B.W., and Churchman, L.S. (2021). Hsf1 activation by proteotoxic stress requires concurrent protein synthesis. Mol Biol Cell 32, 1800-1806.

Vo, M.N., Terrey, M., Lee, J.W., Roy, B., Moresco, J.J., Sun, L., Fu, H., Liu, Q., Weber, T.G., Yates, J.R., 3rd, et al. (2018). ANKRD16 prevents neuron loss caused by an editing-defective tRNA synthetase. Nature 557, 510-515.

Xu, G., Pattamatta, A., Hildago, R., Pace, M.C., Brown, H., and Borchelt, D.R. (2016). Vulnerability of newly synthesized proteins to proteostasis stress. J Cell Sci 129, 1892-1901.

Yonashiro, R., Tahara, E.B., Bengtson, M.H., Khokhrina, M., Lorenz, H., Chen, K.C., Kigoshi-Tansho, Y., Savas, J.N., Yates, J.R., Kay, S.A., et al. (2016). The Rqc2/Tae2 subunit of the ribosome-associated quality control (RQC) complex marks ribosome-stalled nascent polypeptide chains for aggregation. Elife 5, e11794.

Zhang, X., Li, L., Chen, S., Yang, D., Wang, Y., Zhang, X., Wang, Z., and Le, W. (2011). Rapamycin treatment augments motor neuron degeneration in SOD1(G93A) mouse model of amyotrophic lateral sclerosis. Autophagy 7, 412-425. 
bioRxiv preprint doi: https://doi.org/10.1101/2022.01.23.477418; this version posted January 24,2022 . The copyright holder has placed this preprint (which was not certified by peer review) in the Public Domain. It is no longer restricted by copyright. Anyone can legally share, reuse, remix, or adapt this material for any purpose without crediting the original authors.

64 Zhou, C., Slaughter, Brian D., Unruh, Jay R., Guo, F., Yu, Z., Mickey, K., Narkar, A., Ross, Rhonda T., McClain, M.,

65 and Li, R. (2014). Organelle-Based Aggregation and Retention of Damaged Proteins in Asymmetrically Dividing 66 Cells. Cell 159, 530-542.

67

68

69

70 